\title{
SPECIAL HELICES ON EQUIFORM DIFFERENTIAL GEOMETRY OF SPACELIKE CURVES IN MINKOWSKI SPACE-TIME
}

\author{
Fatma BULUT ${ }^{1}$ and Mehmet BEKTAŞ ${ }^{2}$ \\ ${ }^{1}$ Department of Mathematics, Faculty of Arts and Sciences, Bitlis Eren University, 13000 Bitlis, \\ TURKEY \\ ${ }^{2}$ Department of Mathematics, Faculty of Science, Firat University, 23119 Elazı̆̆, TURKEY
}

\begin{abstract}
In this paper, we establish $k$-type helices for equiform differential geometry of spacelike curves in 4-dimensional Minkowski space $\mathrm{E}_{1}^{4}$. Also we obtain $(k, m)$-type slant helices for equiform differential geometry of spacelike curves in Minkowski space-time.
\end{abstract}

\section{INTRODUCTION}

Helices, which are an important subject of the theory of curves in differential geometry, are studied by physicists, engineers and biologists. Helix (or general helix) is described as an in 3-dimensional Euclidean space (or Minkowski) tangent vector field forming a constant angle with a fixed direction of the curve. So, many authors were interested in helices to study it in Euclidean (or Minkowski) 3- and 4-space and they gave new characterizations for an helix. In the 4-dimensional Minkowski space $k$-type slant helices were defined in a study by Ali et al. [1]. In addition, M.Y. Yllmaz and M.Bektaş in [6] defined $(k, m)$-type slant helices in 4-dimensional Euclidean space.

In our study, we establish $k$-type helices and $(k, m)$-type slant helices for equiform differential geometry of spacelike curves in 4-dimensional Minkowski space $\mathrm{E}_{1}^{4}$ and give some new characterizations for these helices.

2020 Mathematics Subject Classification. 53A35.

Keywords and phrases. Equiform Frenet frame, $k$-type helices, $(k, m)$-type slant helices.

\bulut@beu.edu.tr (Corresponding author); mbektas@firat.edu.tr

(D) 0000-0002-7684-6796; 0000-0002-5797-4944. 


\section{Geometric Preliminaries}

Let $E^{4}=\left\{\left(x_{1}, x_{2}, x_{3}, x_{4}\right) \mid x_{1}, x_{2}, x_{3}, x_{4} \in R\right\}$ be a 4-dimensional vector space. For any two vectors $x=\left(x_{1}, x_{2}, x_{3}, x_{4}\right), y=\left(y_{1}, y_{2}, y_{3}, y_{4}\right)$ in $\mathrm{E}^{4}$, the pseudo scalar product of $\mathrm{x}$ and $\mathrm{y}$ is defined by $\langle x, y\rangle=-x_{1} y_{1}+x_{2} y_{2}+x_{3} y_{3}+x_{4} y_{4}$. We call $\left(E^{4},\langle.,\rangle.\right)$ a Minkowski 4 -space and denote it by $E_{1}^{4}$. We say that a vector $x$ in $E_{1}^{4} \backslash\{0\}$ is a spacelike vector, a lightlike vector or a timelike vector if $\langle x, x\rangle$ is positive, zero, negative respectively.

The norm of a vector $x \in E_{1}^{4}$ is defined by $\|x\|=\sqrt{|\langle x, x\rangle|}$. For any two vectors $a, b$ in $E_{1}^{4}$, we say that $a$ is pseudo-perpendicular to $b$ if $\langle a, b\rangle=0$. Let $\alpha: I \subset R \rightarrow E_{1}^{4}$ be an arbitrary curve in $E_{1}^{4}$, we say that a curve $\alpha$ is a spacelike curve if $\langle\dot{\alpha}(t), \dot{\alpha}(t)\rangle>0$ for any $t \in I$. The arclength of a spacelike curve $\gamma$ measured from $\alpha\left(t_{0}\right)\left(t_{0} \in I\right)$ is

$$
s(t)=\int_{t_{0}}^{t}\|\dot{\alpha}(t)\| \mathrm{d} t .
$$

Hence a parameter $s$ is determined such that $\left\|\alpha^{\prime}(s)\right\|=1$, where $\alpha^{\prime}(s)=d \alpha / d s$. Consequently, we say that a spacelike curve $\alpha$ is parameterized by arclength if $\left\|\alpha^{\prime}(s)\right\|=1$. Throughout the rest of this paper $s$ is assumed arclength parameter. For any $x, y, z \in E_{1}^{4}$, we define a vector $x \times y \times z$ by

$$
x \times y \times z=\left|\begin{array}{cccc}
-e_{1} & e_{2} & e_{3} & e_{4} \\
x_{1}^{1} & x_{1}^{2} & x_{1}^{3} & x_{1}^{4} \\
x_{2}^{1} & x_{2}^{2} & x_{2}^{3} & x_{2}^{4} \\
x_{3}^{1} & x_{3}^{2} & x_{3}^{3} & x_{3}^{4}
\end{array}\right|,
$$

where $x_{i}=\left(x_{i}^{1}, x_{i}^{2}, x_{i}^{3}, x_{i}^{4}\right)$. Let $\alpha: I \longrightarrow E_{1}^{4}$ be a spacelike curve in $E_{1}^{4}$. Therefore we can construct a pseudo-orthogonal frame $\left\{\mathbf{t}(s), \mathbf{n}(s), \mathbf{b}_{\mathbf{1}}(s), \mathbf{b}_{\mathbf{2}}(s)\right\}$, which satisfies the following Frenet-Serret type formula of $\mathrm{E}_{1}^{4}$ along $\alpha$.

$$
\left[\begin{array}{c}
\mathbf{t} \\
\mathbf{n} \\
\mathbf{b}_{\mathbf{1}} \\
\mathbf{b}_{\mathbf{2}}
\end{array}\right]^{\prime}=\left[\begin{array}{cccc}
0 & \kappa_{1} & 0 & 0 \\
\mu_{1} \kappa_{1} & 0 & \mu_{2} \kappa_{2} & 0 \\
0 & \mu_{3} \kappa_{2} & 0 & \mu_{4} \kappa_{3} \\
0 & 0 & \mu_{5} \kappa_{3} & 0
\end{array}\right]\left[\begin{array}{c}
\mathbf{t} \\
\mathbf{n} \\
\mathbf{b}_{\mathbf{1}} \\
\mathbf{b}_{\mathbf{2}}
\end{array}\right],
$$

where $\kappa_{1}, \kappa_{2}$ and $\kappa_{3}$ are respectively, first, second and third curvature of the spacelike curve $\alpha$ and we have

$$
\begin{aligned}
& \kappa_{1}(s)=\left\|\alpha^{\prime \prime}(s)\right\|, \\
& \mathbf{n}(s)=\frac{\alpha^{\prime \prime}(s)}{\kappa_{1}(s)}, \\
& \mathbf{b}_{1}(s)=\frac{\mathbf{n}^{\prime}(s)+\mu_{1} \kappa_{1}(s) \mathbf{t}(s)}{\left\|\mathbf{n}^{\prime}(s)+\mu_{1} \kappa_{1}(s) \mathbf{t}(s)\right\|},
\end{aligned}
$$




$$
\mathbf{b}_{\mathbf{2}}(s)=\mathbf{t}(s) \times \mathbf{n}(s) \times \mathbf{b}_{\mathbf{1}}(s) .
$$

Denote by $\left\{\mathbf{t}(s), \mathbf{n}(s), \mathbf{b}_{\mathbf{1}}(s), \mathbf{b}_{\mathbf{2}}(s)\right\}$ the moving Frenet frame along the spacelike curve $\alpha$, where $s$ is a pseudo arclength parameter $[1,2,3,5,7]$.

\section{Equiform Differential Geometry of Curves}

\subsection{Spacelike Curves:}

Definition 3.1. Unless otherwise stated, we use the same terminology such as $[2,4]$. Let $\alpha: I \longrightarrow E_{1}^{4}$ be a spacelike curve. We define the equiform parameter of $\alpha(s)$ by

$$
\sigma=\int \frac{d s}{\rho}=\int \kappa_{1} d s
$$

where $\rho=\frac{1}{\kappa_{1}}$ is the radius of curvature of the curve $\alpha$.

It follows

$$
\frac{d s}{d \sigma}=\rho
$$

Let $h$ be a homothety with the center in the origin and the coefficient $\lambda$. If we put $\alpha^{*}=h(\alpha)$ then it follows

$$
s^{*}=\lambda s, \text { and } \rho^{*}=\lambda \rho,
$$

where $s^{*}$ is the arclength parameter of $\alpha^{*}$ and $\rho^{*}$ the radius of curvature of $\alpha^{*}$. Hence $\alpha$ is an equiform invariant parameter of $\alpha$.

Notation 3.1. Let us note that $\kappa_{1}, \kappa_{2}$ and $\kappa_{3}$ are not invariants of the homothety group, it follows $\kappa_{1}^{*}=\frac{1}{\lambda} \kappa_{1}, \kappa_{2}^{*}=\frac{1}{\lambda} \kappa_{2}$ and $\kappa_{3}^{*}=\frac{1}{\lambda} \kappa_{3}$. The vector

$$
\mathbf{V}_{1}=\frac{d \alpha(s)}{d \sigma}
$$

is called a tangent vector of the curve $\alpha$ in the equiform geometry. From (5) and (7), we get

$$
\mathbf{V}_{1}=\frac{d \alpha(s)}{d \sigma}=\rho \frac{d \alpha(s)}{d s}=\rho \mathbf{t}
$$

Furthermore, we define the tri-normals by

$$
\mathbf{V}_{2}=\rho \mathbf{n}, \quad \mathbf{V}_{3}=\rho \mathbf{b}_{\mathbf{1}}, \quad \mathbf{V}_{4}=\rho \mathbf{b}_{\mathbf{2}} .
$$

It is easy to check that the tetrahedron $\left\{\mathbf{V}_{1}, \mathbf{V}_{2}, \mathbf{V}_{3}, \mathbf{V}_{4}\right\}$ is an equiform invariant tetrahedron of the curve $\alpha$. Now, we will find the derivatives of these vectors with respect to $\sigma$ using by (5), (7) and (9) as follows:

$$
\mathbf{V}_{1}^{\prime}=\frac{d}{d \sigma}\left(\mathbf{V}_{1}\right)=\rho \frac{d}{d s}(\rho \mathbf{t})=\dot{\boldsymbol{\rho}} \mathbf{V}_{\mathbf{1}}+\mathbf{V}_{\mathbf{2}}
$$


where the derivative with respect to the arclength $s$ is denoted by a dot and respect to $\sigma$ by a dash. Similarly, we obtain

$$
\begin{aligned}
& \mathbf{V}_{2}^{\prime}=\frac{d}{d \sigma}\left(\mathbf{V}_{2}\right)=\rho \frac{d}{d s}(\rho \mathbf{n})=\boldsymbol{\mu}_{\mathbf{1}} \mathbf{V}_{\mathbf{1}}+\dot{\boldsymbol{\rho}} \mathbf{V}_{\mathbf{2}}+\boldsymbol{\mu}_{\mathbf{2}}\left(\frac{\boldsymbol{\kappa}_{\mathbf{2}}}{\boldsymbol{\kappa}_{\mathbf{1}}}\right) \mathbf{V}_{\mathbf{3}} \\
& \mathbf{V}_{3}^{\prime}=\frac{d}{d \sigma}\left(\mathbf{V}_{3}\right)=\rho \frac{d}{d s}\left(\rho \mathbf{b}_{\mathbf{1}}\right)=\boldsymbol{\mu}_{\mathbf{3}}\left(\frac{\boldsymbol{\kappa}_{\mathbf{2}}}{\boldsymbol{\kappa}_{\mathbf{1}}}\right) \mathbf{V}_{\mathbf{2}}+\dot{\boldsymbol{\rho}} \mathbf{V}_{\mathbf{3}}+\boldsymbol{\mu}_{\mathbf{4}}\left(\frac{\boldsymbol{\kappa}_{\mathbf{3}}}{\boldsymbol{\kappa}_{\mathbf{1}}}\right) \mathbf{V}_{\mathbf{4}} \\
& \mathbf{V}_{4}^{\prime}=\frac{d}{d \sigma}\left(\mathbf{V}_{4}\right)=\rho \frac{d}{d s}\left(\rho \mathbf{b}_{\mathbf{2}}\right)=\boldsymbol{\mu}_{\mathbf{5}}\left(\frac{\boldsymbol{\kappa}_{\mathbf{3}}}{\boldsymbol{\kappa}_{\mathbf{1}}}\right) \mathbf{V}_{\mathbf{3}}+\dot{\boldsymbol{\rho}} \mathbf{V}_{\mathbf{4}}
\end{aligned}
$$

Definition 3.2. The functions $\mathbf{K}_{i}: I \longrightarrow R(i=1,2,3)$ defined by

$$
\mathbf{K}_{1}=\dot{\rho}, \mathbf{K}_{2}=\frac{\kappa_{2}}{\kappa_{1}}, \mathbf{K}_{3}=\frac{\kappa_{3}}{\kappa_{1}}
$$

are called $i^{\text {th }}$ equiform curvatures of the curve $\alpha$.

These functions $\mathbf{K}_{i}$ are differential invariant of the group of equiform transformations, too. Therefore, the formulas analogous to famous the Frenet formulas in the equiform geometry of the Minkowski space $E_{1}^{4}$ have the following form:

$$
\begin{aligned}
\mathbf{V}_{1}^{\prime} & =\mathbf{K}_{1} \mathbf{V}_{1}+\mathbf{V}_{2}, \\
\mathbf{V}_{2}^{\prime} & =\mu_{1} \mathbf{V}_{1}+\mathbf{K}_{1} \mathbf{V}_{2}+\mu_{2} \mathbf{K}_{2} \mathbf{V}_{3}, \\
\mathbf{V}_{3}^{\prime} & =\mu_{3} \mathbf{K}_{2} \mathbf{V}_{2}+\mathbf{K}_{1} \mathbf{V}_{3}+\mu_{4} \mathbf{K}_{3} \mathbf{V}_{4}, \\
\mathbf{V}_{4}^{\prime} & =\mu_{5} \mathbf{K}_{3} \mathbf{V}_{3}+\mathbf{K}_{1} \mathbf{V}_{4} .
\end{aligned}
$$

Notation 3.2. The equiform parameter $\sigma=\int \kappa_{1}(s) d s$ for closed curves is called the total curvature, and it plays an important role in global differential geometry of the Euclidean space. Also, the functions $\frac{\kappa_{2}}{\kappa_{1}}$ and $\frac{\kappa_{3}}{\kappa_{1}}$ have been already known as conical curvatures and they also have interesting geometric interpretation.

Because of the equiform Frenet formulas (12), the following equalities regarding equiform curvatures can be given

$$
\begin{aligned}
& \mathbf{K}_{1}=\frac{1}{\rho^{2}}\left\langle\mathbf{V}_{j}^{\prime}, \mathbf{V}_{j}\right\rangle ;(j=1,2,3,4), \\
& \mathbf{K}_{2}=\frac{1}{\mu_{2} \rho^{2}}\left\langle\mathbf{V}_{2}^{\prime}, \mathbf{V}_{3}\right\rangle=\frac{1}{\mu_{3} \rho^{2}}\left\langle\mathbf{V}_{3}^{\prime}, \mathbf{V}_{2}\right\rangle, \\
& \mathbf{K}_{3}=\frac{1}{\mu_{4} \rho^{2}}\left\langle\mathbf{V}_{3}^{\prime}, \mathbf{V}_{4}\right\rangle=\frac{1}{\mu_{5} \rho^{2}}\left\langle\mathbf{V}_{4}^{\prime}, \mathbf{V}_{3}\right\rangle .
\end{aligned}
$$

Definition 3.3. Let $\alpha$ be a spacelike curve in $E_{1}^{4}$ with equiform Frenet frame $\left\{\mathbf{V}_{1}, \mathbf{V}_{2}, \mathbf{V}_{3}, \mathbf{V}_{4}\right\}$. If there exists a non-zero constant vector field $U$ in $E_{1}^{4}$ such that $<\mathbf{V}_{i}, U>=$ constant for $1 \leq i \leq 4$, then $\alpha$ is said to be a $k$-type slant helix and $U$ is called the slope axis of $\alpha$. 
Theorem 3.1. Let $\alpha$ be a spacelike curve with Frenet formulas in equiform geometry of the Minkowski space $E_{1}^{4}$. Then, if the curve $\alpha$ is a 1-type helix (or general helix), then we have

$$
\left\langle\mathbf{V}_{2}, U\right\rangle=-\mathbf{K}_{1} c
$$

where $c$ is a constant.

Proof. Assume that $\alpha$ is a 1-type helix. Then for a constant field $U$ such that $\left\langle\mathbf{V}_{1}, U\right\rangle=c$ is a constant. Differentiating this equation with respect to $\sigma$, we get

$$
\left\langle\mathbf{V}_{1}^{\prime}, U\right\rangle=0,
$$

and using equiform Frenet equations, we find

$$
\mathbf{K}_{1}\left\langle\mathbf{V}_{1}, U\right\rangle+\left\langle\mathbf{V}_{2}, U\right\rangle=0
$$

and using $\left\langle\mathbf{V}_{1}, U\right\rangle=c$,

$$
\mathbf{K}_{1} c+\left\langle\mathbf{V}_{2}, U\right\rangle=0
$$

From (15), it is written as follows:

$$
\left\langle\mathbf{V}_{2}, U\right\rangle=-\mathbf{K}_{1} c,
$$

thus, the proof is completed.

Theorem 3.2. Let $\alpha$ be a spacelike curve with Frenet formulas in equiform geometry of the Minkowski space $E_{1}^{4}$. Then, if the curve $\alpha$ is a 2-type helix, then we have

$$
\mu_{1}\left\langle\mathbf{V}_{1}, U\right\rangle+\mu_{2} \mathbf{K}_{2}\left\langle\mathbf{V}_{3}, U\right\rangle=-\mathbf{K}_{1} c_{1}
$$

where $c_{1}$ is a constant.

Proof. If the curve $\alpha$ is a 2-type helix. Therefore for a constant field $U$ such that $\left\langle\mathbf{V}_{2}, U\right\rangle=c_{1}$ is a constant. Differentiating this equation with respect to $\sigma$, we get

$$
\left\langle\mathbf{V}_{2}^{\prime}, U\right\rangle=0,
$$

and using equiform Frenet equations, we have

$$
\mu_{1}\left\langle\mathbf{V}_{1}, U\right\rangle+\mathbf{K}_{1}\left\langle\mathbf{V}_{2}, U\right\rangle+\mu_{2} \mathbf{K}_{2}\left\langle\mathbf{V}_{3}, U\right\rangle=0
$$

and using $\left\langle\mathbf{V}_{2}, U\right\rangle=c_{1}$, we find

$$
\mu_{1}\left\langle\mathbf{V}_{1}, U\right\rangle+\mathbf{K}_{1} c_{1}+\mu_{2} \mathbf{K}_{2}\left\langle\mathbf{V}_{3}, U\right\rangle=0
$$

From (17), we obtain

$$
\mu_{1}\left\langle\mathbf{V}_{1}, U\right\rangle+\mu_{2} \mathbf{K}_{2}\left\langle\mathbf{V}_{3}, U\right\rangle=-\mathbf{K}_{1} c_{1} .
$$

The proof is completed.

Theorem 3.3. Let $\alpha$ be a spacelike curve with Frenet formulas in equiform geometry of the Minkowski space $E_{1}^{4}$. In that case, if the curve $\alpha$ is a 3-type helix, then we have

$$
\mu_{3} \mathbf{K}_{2}\left\langle\mathbf{V}_{2}, U\right\rangle+\mu_{4} \mathbf{K}_{3}\left\langle\mathbf{V}_{4}, U\right\rangle=-\mathbf{K}_{1} c_{2},
$$

where $c_{2}$ is a constant. 
Proof. If the curve $\alpha$ is a 3 -type helix. Thus, for a constant field $U$ such that

$$
\left\langle\mathbf{V}_{3}, U\right\rangle=c_{2}
$$

is a constant. Differentiating this equation with respect to $\sigma$, we get

$$
\left\langle\mathbf{V}_{3}^{\prime}, U\right\rangle=0
$$

and using equiform Frenet equations, we have

$$
\mu_{3} \mathbf{K}_{2}\left\langle\mathbf{V}_{2}, U\right\rangle+\mathbf{K}_{1}\left\langle\mathbf{V}_{3}, U\right\rangle+\mu_{4} \mathbf{K}_{3}\left\langle\mathbf{V}_{4}, U\right\rangle=0
$$

and by setting (19) in (20), we can write

$$
\mu_{3} \mathbf{K}_{2}\left\langle\mathbf{V}_{2}, U\right\rangle+\mathbf{K}_{1} c_{2}+\mu_{4} \mathbf{K}_{3}\left\langle\mathbf{V}_{4}, U\right\rangle=0
$$

and from the last equation, we find

$$
\mu_{3} \mathbf{K}_{2}\left\langle\mathbf{V}_{2}, U\right\rangle+\mu_{4} \mathbf{K}_{3}\left\langle\mathbf{V}_{4}, U\right\rangle=-\mathbf{K}_{1} c_{2},
$$

the proof is completed.

Theorem 3.4. Let $\alpha$ be a spacelike curve with Frenet formulas in equiform geometry of the Minkowski space $E_{1}^{4}$. Then, if the curve $\alpha$ is a 4-type helix, in that case, we have

$$
\left\langle\mathbf{V}_{3}, U\right\rangle=-\frac{\mathbf{K}_{1}}{\mathbf{K}_{3} \mu_{5}} c_{3}
$$

where $c_{3}$ is a constant.

Proof. If the curve $\alpha$ is a 4 -type helix. Then for a constant field $U$ such that

$$
\left\langle\mathbf{V}_{4}, U\right\rangle=c_{3},
$$

is a constant. By differentiating of this last equation with respect to $\sigma$, we get

$$
\left\langle\mathbf{V}_{4}^{\prime}, U\right\rangle=0,
$$

and using equiform Frenet equations, we obtain

$$
\left\langle\mu_{5} \mathbf{K}_{3} \mathbf{V}_{3}+\mathbf{K}_{1} \mathbf{V}_{4}, U\right\rangle=0 .
$$

From (24), we get

$$
\mu_{5} \mathbf{K}_{3}\left\langle\mathbf{V}_{3}, U\right\rangle+\mathbf{K}_{1}\left\langle\mathbf{V}_{4}, U\right\rangle=0 .
$$

Substituting (23) in (25), we obtain

$$
\left\langle\mathbf{V}_{3}, U\right\rangle=-\frac{\mathbf{K}_{1}}{\mathbf{K}_{3} \mu_{5}} c_{3} .
$$

The proof is completed. 


\section{4. $(k, m)$-type slant helices in $\mathbf{E}_{1}^{4}$}

In this section, we will define $(k, m)$ type slant helices for spacelike curve with equiform Frenet frame in $\mathrm{E}_{1}^{4}$ such as [6].

Definition 4.1. Let $\alpha$ be a spacelike curve in $E_{1}^{4}$ with equiform Frenet frame $\left\{\mathbf{V}_{1}, \mathbf{V}_{2}, \mathbf{V}_{3}, \mathbf{V}_{4}\right\}$. We call $\alpha$ is a $(k, m)$ - type slant helix if there exists a nonzero constant vector field $U \in E_{1}^{4}$ satisfies $\left\langle\mathbf{V}_{k}, U\right\rangle=c_{1}\left(c_{1}\right.$ is a constant) and $\left\langle\mathbf{V}_{m}, U\right\rangle=c_{2}$ ( $c_{2}$ is a constant) for $1 \leq k, m \leq 4, k \neq m$. The constant vector $U$ is on axis of $\alpha$.

Theorem 4.1. If the curve $\alpha$ is a $(1,2)$-type slant helix in $E_{1}^{4}$, then we have

$$
\left\langle\mathbf{V}_{3}, U\right\rangle=-\frac{\mu_{1} c_{1}+\mathbf{K}_{1} c_{2}}{\mu_{2} \mathbf{K}_{2}},
$$

and

$$
\mathbf{K}_{1}=-\frac{c_{2}}{c_{1}} \text { is a constant. }
$$

Proof. If the curve $\alpha$ is a $(1,2)$-type slant helix in $\mathrm{E}_{1}^{4}$, then for a constant field $U$. We can write

$$
\left\langle\mathbf{V}_{1}, U\right\rangle=c_{1}
$$

and

$$
\left\langle\mathbf{V}_{2}, U\right\rangle=c_{2}
$$

is a constant. Differentiating (26) and (27) with respect to $\sigma$, we have that

$$
\left\langle\mathbf{V}_{1}^{\prime}, U\right\rangle=0
$$

and

$$
\left\langle\mathbf{V}_{2}^{\prime}, U\right\rangle=0
$$

Using equiform Frenet equations, the following equations can be obtained:

$$
\begin{gathered}
\mathbf{K}_{1}\left\langle\mathbf{V}_{1}, U\right\rangle+\left\langle\mathbf{V}_{2}, U\right\rangle=0 \\
\mu_{1}\left\langle\mathbf{V}_{1}, U\right\rangle+\mathbf{K}_{1}\left\langle\mathbf{V}_{2}, U\right\rangle+\mu_{2} \mathbf{K}_{2}\left\langle\mathbf{V}_{3}, U\right\rangle=0 .
\end{gathered}
$$

By setting (26) and (27) in (28), we find

$$
\mathbf{K}_{1} c_{1}+c_{2}=0
$$

and substituting (26) and (27) in (28), we obtain

$$
\mu_{1} c_{1}+\mathbf{K}_{1} c_{2}+\mu_{2} \mathbf{K}_{2}\left\langle\mathbf{V}_{3}, U\right\rangle=0
$$

Finally, we have the following equations:

$$
\begin{aligned}
\mathbf{K}_{1} & =-\frac{c_{2}}{c_{1}}, \\
\left\langle\mathbf{V}_{3}, U\right\rangle & =-\frac{\mu_{1} c_{1}+\mathbf{K}_{1} c_{2}}{\mu_{2} \mathbf{K}_{2}} .
\end{aligned}
$$

The proof is completed. 
Theorem 4.2. If the curve $\alpha$ is a $(1,3)$-type slant helix in $E_{1}^{4}$, then there exists a constant such that

$$
\left\langle\mathbf{V}_{4}, U\right\rangle=\frac{\mu_{3} \mathbf{K}_{2} \mathbf{K}_{1} c_{1}-\mathbf{K}_{1} c_{3}}{\mu_{4} \mathbf{K}_{3}} .
$$

where $c_{1}$ and $c_{3}$ are constant.

Proof. If the curve $\alpha$ is a $(1,3)$-type slant helix in $\mathrm{E}_{1}^{4}$, then for a constant field $U$. We can write

$$
\left\langle\mathbf{V}_{1}, U\right\rangle=c_{1}
$$

and

$$
\left\langle\mathbf{V}_{3}, U\right\rangle=c_{3}
$$

is a constant. Differentiating (33) and (34) with respect to $\sigma$, we get

$$
\left\langle\mathbf{V}_{1}^{\prime}, U\right\rangle=0,
$$

and

$$
\left\langle\mathbf{V}_{3}^{\prime}, U\right\rangle=0 .
$$

Using equiform Frenet equations, we have

$$
\begin{gathered}
\mathbf{K}_{1}\left\langle\mathbf{V}_{1}, U\right\rangle+\left\langle\mathbf{V}_{2}, U\right\rangle=0 \\
\mu_{3} \mathbf{K}_{2}\left\langle\mathbf{V}_{2}, U\right\rangle+\mathbf{K}_{1}\left\langle\mathbf{V}_{3}, U\right\rangle+\mu_{4} \mathbf{K}_{3}\left\langle\mathbf{V}_{4}, U\right\rangle=0 .
\end{gathered}
$$

By setting (33) in (35), we obtain

$$
\mathbf{K}_{1} c_{1}+\left\langle\mathbf{V}_{2}, U\right\rangle=0 .
$$

From (37), we find as follows:

$$
\left\langle\mathbf{V}_{2}, U\right\rangle=-\mathbf{K}_{1} c_{1} .
$$

Substituting (34) and (38) in (36), we find

$$
\left\langle\mathbf{V}_{4}, U\right\rangle=\frac{\mu_{3} \mathbf{K}_{2} \mathbf{K}_{1} c_{1}-\mathbf{K}_{1} c_{3}}{\mu_{4} \mathbf{K}_{3}} .
$$

The proof is completed.

Theorem 4.3. If the curve $\alpha$ is a $(1,4)$-type slant helix in $E_{1}^{4}$, then there exists a constant such that

$$
\left\langle\mathbf{V}_{2}, U\right\rangle=-\mathbf{K}_{1} c_{1}
$$

and

$$
\left\langle\mathbf{V}_{3}, U\right\rangle=-\frac{\mathbf{K}_{1} c_{4}}{\mu_{5} \mathbf{K}_{3}}
$$


Proof. If the curve $\alpha$ is a $(1,4)$-type slant helix in $\mathrm{E}_{1}^{4}$, then for a constant field $U$. We can write

$$
\left\langle\mathbf{V}_{1}, U\right\rangle=c_{1}
$$

and

$$
\left\langle\mathbf{V}_{4}, U\right\rangle=c_{4}
$$

is a constant. Differentiating (39) and (40) with respect to $\sigma$, we get

$$
\left\langle\mathbf{V}_{1}^{\prime}, U\right\rangle=0
$$

and it follows

$$
\left\langle\mathbf{V}_{4}^{\prime}, U\right\rangle=0
$$

Using equiform Frenet equations, we have

$$
\mathbf{K}_{1}\left\langle\mathbf{V}_{1}, U\right\rangle+\left\langle\mathbf{V}_{2}, U\right\rangle=0
$$

and

$$
\mu_{5} \mathbf{K}_{3}\left\langle\mathbf{V}_{3}, U\right\rangle+\mathbf{K}_{1}\left\langle\mathbf{V}_{4}, U\right\rangle=0
$$

By setting (39) in (41), we obtain as below:

$$
\mathbf{K}_{1} c_{1}+\left\langle\mathbf{V}_{2}, U\right\rangle=0 .
$$

Substituting (40) in (42), we can write

$$
\mu_{5} \mathbf{K}_{3}\left\langle\mathbf{V}_{3}, U\right\rangle+\mathbf{K}_{1} c_{4}=0 .
$$

From (43) and (44), we get

and

$$
\left\langle\mathbf{V}_{2}, U\right\rangle=-\mathbf{K}_{1} c_{1}
$$

The proof is completed.

$$
\left\langle\mathbf{V}_{3}, U\right\rangle=-\frac{\mathbf{K}_{1} c_{4}}{\mu_{5} \mathbf{K}_{3}}
$$

Theorem 4.4. If the curve $\alpha$ is a $(2,3)$-type slant helix in $E_{1}^{4}$, then there exist constants such that

and

$$
\left\langle\mathbf{V}_{1}, U\right\rangle=-\frac{\mathbf{K}_{1} c_{2}+\mu_{2} \mathbf{K}_{2} c_{3}}{\mu_{1}},
$$

$$
\left\langle\mathbf{V}_{4}, U\right\rangle=-\frac{\mu_{3} \mathbf{K}_{2} c_{2}+\mathbf{K}_{1} c_{3}}{\mu_{4} \mathbf{K}_{3}} .
$$

Proof. If the curve $\alpha$ is a (2,3)-type slant helix in $\mathrm{E}_{1}^{4}$, thus for a constant field $U$. We can write as below:

$$
\left\langle\mathbf{V}_{2}, U\right\rangle=c_{2}
$$

is a constant and

$$
\left\langle\mathbf{V}_{3}, U\right\rangle=c_{3}
$$

is a constant. Differentiating (47) and (48) with respect to $\sigma$, we get

$$
\left\langle\mathbf{V}_{2}^{\prime}, U\right\rangle=0,
$$


and

$$
\left\langle\mathbf{V}_{3}^{\prime}, U\right\rangle=0
$$

Using equiform Frenet formulas, we have the following equations:

$$
\begin{gathered}
\mu_{1}\left\langle\mathbf{V}_{1}, U\right\rangle+\mathbf{K}_{1}\left\langle\mathbf{V}_{2}, U\right\rangle+\mu_{2} \mathbf{K}_{2}\left\langle\mathbf{V}_{3}, U\right\rangle=0, \\
\mu_{3} \mathbf{K}_{2}\left\langle\mathbf{V}_{2}, U\right\rangle+\mathbf{K}_{1}\left\langle\mathbf{V}_{3}, U\right\rangle+\mu_{4} \mathbf{K}_{3}\left\langle\mathbf{V}_{4}, U\right\rangle=0 .
\end{gathered}
$$

Substituting (47) and (48) in (49), we can write

$$
\left\langle\mathbf{V}_{1}, U\right\rangle=-\frac{\mathbf{K}_{1} c_{2}+\mu_{2} \mathbf{K}_{2} c_{3}}{\mu_{1}},
$$

and by setting (47) and (48) in (50), we obtain

$$
\left\langle\mathbf{V}_{4}, U\right\rangle=-\frac{\mu_{3} \mathbf{K}_{2} c_{2}+\mathbf{K}_{1} c_{3}}{\mu_{4} \mathbf{K}_{3}} .
$$

The proof is completed.

Theorem 4.5. If the curve $\alpha$ is a $(2,4)$-type slant helix in $E_{1}^{4}$, then there exists constant such that

$$
\left\langle\mathbf{V}_{1}, U\right\rangle=\frac{\mu_{2} \mathbf{K}_{2} \mathbf{K}_{1} c_{4}-\mu_{5} \mathbf{K}_{3} \mathbf{K}_{1} c_{2}}{\mu_{1} \mu_{5} \mathbf{K}_{3}},
$$

where $c_{2}$ and $c_{4}$ are constants.

Proof. If the curve $\alpha$ is a $(2,4)$-type slant helix in $\mathrm{E}_{1}^{4}$, then for a constant field $U$. We can write the following equations:

$$
\left\langle\mathbf{V}_{2}, U\right\rangle=c_{2}
$$

and

$$
\left\langle\mathbf{V}_{4}, U\right\rangle=c_{4}
$$

is a constant. By differentiating (51) and (52) with respect to $\sigma$, we get the following equations:

and

$$
\left\langle\mathbf{V}_{2}^{\prime}, U\right\rangle=0
$$

$$
\left\langle\mathbf{V}_{4}^{\prime}, U\right\rangle=0
$$

Using equiform Frenet equations, we have as below:

$$
\begin{gathered}
\mu_{1}\left\langle\mathbf{V}_{1}, U\right\rangle+\mathbf{K}_{1}\left\langle\mathbf{V}_{2}, U\right\rangle+\mu_{2} \mathbf{K}_{2}\left\langle\mathbf{V}_{3}, U\right\rangle=0 \\
\mu_{5} \mathbf{K}_{3}\left\langle\mathbf{V}_{3}, U\right\rangle+\mathbf{K}_{1}\left\langle\mathbf{V}_{4}, U\right\rangle=0
\end{gathered}
$$

Using (52), in the last equation, we get

$$
\left\langle\mathbf{V}_{3}, U\right\rangle=-\frac{\mathbf{K}_{1} c_{4}}{\mu_{5} \mathbf{K}_{3}}
$$

Substituting (51) and (55) in (53), we obtain

$$
\left\langle\mathbf{V}_{1}, U\right\rangle=\frac{\mu_{2} \mathbf{K}_{2} \mathbf{K}_{1} c_{4}-\mu_{5} \mathbf{K}_{3} \mathbf{K}_{1} c_{2}}{\mu_{1} \mu_{5} \mathbf{K}_{3}},
$$


the proof is completed.

Theorem 4.6. If the curve $\alpha$ is a $(3,4)$-type slant helix in $E_{1}^{4}$, then we have

$$
\left\langle\left\langle\mathbf{V}_{2}, U\right\rangle=\frac{\mathbf{K}_{3}}{\mathbf{K}_{2}} \frac{\left(\mu_{5} c_{3}^{2}-\mu_{4} c_{4}^{2}\right)}{\mu_{3} c_{4}}\right.
$$

and

$$
\mathbf{K}_{1}=-\mu_{5} \mathbf{K}_{3} \frac{c_{3}}{c_{4}} .
$$

Proof. If the curve $\alpha$ is a $(3,4)$-type slant helix in $\mathrm{E}_{1}^{4}$, then for a constant field $U$. We can write

$$
\left\langle\mathbf{V}_{3}, U\right\rangle=c_{3}
$$

is a constant and

$$
\left\langle\mathbf{V}_{4}, U\right\rangle=c_{4}
$$

is a constant. By differentiating (57) and (58) with respect to $\sigma$, we get

$$
\left\langle\mathbf{V}_{3}^{\prime}, U\right\rangle=0
$$

and it follows

$$
\left\langle\mathbf{V}_{4}^{\prime}, U\right\rangle=0
$$

Using equiform Frenet formulas, we get as follows:

$$
\begin{gathered}
\mu_{3} \mathbf{K}_{2}\left\langle\mathbf{V}_{2}, U\right\rangle+\mathbf{K}_{1}\left\langle\mathbf{V}_{3}, U\right\rangle+\mu_{4} \mathbf{K}_{3}\left\langle\mathbf{V}_{4}, U\right\rangle=0 \\
\mu_{5} \mathbf{K}_{3}\left\langle\mathbf{V}_{3}, U\right\rangle+\mathbf{K}_{1}\left\langle\mathbf{V}_{4}, U\right\rangle=0
\end{gathered}
$$

By setting (57) and (58) in (60), we have the following equation:

$$
\mathbf{K}_{1}=-\mu_{5} \mathbf{K}_{3} \frac{c_{3}}{c_{4}}
$$

and substituting (57) and (58) in (59), we obtain

$$
\left\langle\mathbf{V}_{2}, U\right\rangle=-\frac{\mathbf{K}_{1} c_{3}}{\mu_{3} \mathbf{K}_{2}}-\frac{\mu_{4} \mathbf{K}_{3} c_{4}}{\mu_{3} \mathbf{K}_{2}}
$$

Using (61), in the last equation, we find

$$
\left\langle\mathbf{V}_{2}, U\right\rangle=\frac{\mathbf{K}_{3}}{\mathbf{K}_{2}} \frac{\left(\mu_{5} c_{3}^{2}-\mu_{4} c_{4}^{2}\right)}{\mu_{3} c_{4}} .
$$

The proof is completed. 


\section{REFERENCES}

[1] Ali, A. T., López, R., Turgut, M., k-type partially null and pseudo null slant helices in Minkowski 4-space, Math.Commun., 17 (2012), 93-103.

[2] Abdel-Aziz, H.S., Saad, M.K. Abdel-Salam, A.A., Equiform Differential Geometry of Curves in Minkowski Space-Time, arXiv.org/math/ arXiv:1501.02283.

[3] İlarslan, K., Nešović, E., Spacelike and Timelike Normal Curves in Minkowski Space-Time, Publications de L'Institut Mathematique, Nouvelle série, tome 85(99) (2009), 111-118.

[4] Aydın, M.E., Ergüt, M., The equiform differential geometry of curves in 4-dimensional Galilean space G4, Stud. Univ. Babe, s-Bolyai Math., 58(3) (2013), 399-406.

[5] Turgut, M., Yilmaz, S., Characterizations of Some Special Space-like Curves in Minkowski Space-time, International J.Math. Combin., 2 (2008), 17-22.

[6] Yılmaz, M.Y., Bektaş, M., Slant helices of $(k, m)$-type in $\mathrm{E}^{4}$, Acta Univ. Sapientiae, Mathematica, 10(2) (2018), 395-401.

[7] Yilmaz, S., Turgut, M., On the characterizations of inclined curves in Minkowski space-time $\mathrm{E}_{1}^{4}$, International Mathematical Forum, 3(16) (2008), 783-792. 